\title{
Dramatic Resolution of Recalcitrant Cystoid Macular Edema after Concurrent Intravitreal Injection of Bevacizumab and Triamcinolone
}

\author{
Kakarla V. Chalam ${ }^{1}$, Suzie Gasparian ${ }^{1}$, Moises Enghelberg ${ }^{1}$ \\ ${ }^{1}$ Department of Ophthalmology, Loma Linda University School of Medicine, Loma Linda, California, USA
}

Epub: May 20, 2020

\begin{abstract}
Background: Cystoid macular edema (CME), a common complication of branch retinal vein occlusion (BRVO), is associated with a significant vision loss. Anti-vascular endothelial growth factor (anti-VEGF) therapy is the gold standard of treatment, while grid macular photocoagulation has also been used as an adjuvant in patients with CME secondary to BRVO. More recent efforts were successful by the use of intravitreal triamcinolone acetonide. We proposed a concurrent use of intravitreal triamcinolone acetonide and intravitreal bevacizumab in the treatment of CME secondary to BRVO.

Case presentation: We described an 82-year-old female with a BRVO in the right eye who developed associated CME. Repeated injections of intravitreal bevacizumab and modified grid macular laser treatment were ineffective. A concurrent treatment with intravitreal bevacizumab and triamcinolone acetonide resulted in complete and dramatic resolution of CME with a favorable visual outcome. Optical Coherence Tomography (OCT) demonstrated a significant decrease in central subfield thickness (CST) from $764 \mu \mathrm{m}$ to $253 \mu \mathrm{m}$, without any post-procedure complications or recurrence of macular edema with complete recovery of visual acuity at 6-month follow-up.

Conclusion: Early concurrent treatment with intravitreal anti-VEGF therapy (e.g. intravitreal bevacizumab) and intravitreal triamcinolone acetonide is likely to be more effective than intravitreal anti-VEGF agents alone or grid macular photocoagulation in the management of CME associated with BRVO.
\end{abstract}

\section{KEY WORDS}

Branch Retinal Vein Occlusion; Cystoid Macular Edema; Vascular Endothelial Growth Factor; Triamcinolone Acetonide; Grid Focal Macular Laser; Bevacizumab; Optical Coherence Tomography.

Copyright (C) 2020, Med Hypothesis Discov Innov Ophthalmol. This is an open-access article distributed under the terms of the Creative Commons Attribution-NonCommercial 4.0 International License (http://creativecommons.org/licenses/by-nc/4.0/) which permits copy and redistribute the material just in noncommercial usages, provided the original work is properly cited.

Correspondence to: K.V. Chalam, Professor and Director of Retina, Loma Linda University School of Medicine, 11370 Anderson Street, Suite 2025, Loma Linda, CA, USA. E-mail: kvchalam@llu.edu

How to cite this article: Chalam KV, Gasparian S, Enghelberg M, Dramatic Resolution of Recalcitrant Cystoid Macular Edema after Concurrent Intravitreal Injection of Bevacizumab and Triamcinolone. Med Hypothesis Discov Innov Ophthalmol. 2020; 9(3): 159-163

\section{INTRODUCTION}

Branch retinal vein occlusion (BRVO), the second most common cause of retinal vascular disease, is a frequent cause of vision loss, which could impact quality of life to a great extent [1]. Classic clinical features include sectoral retinal hemorrhages and dilated, tortuous retinal veins along with cotton-wool spots in the distribution of the occluded vessel [2].
BRVO causes both retinal ischemia and inflammation [3]. Release of inflammatory mediators such as interleukin-1 (IL-1), interleukin-6 (IL-6), monocyte chemoattractant protein-1 (MCP-1), intracellular adhesion molecule-1 (ICAM-1), and vascular endothelial growth factor (VEGF) result in an increase in vascular permeability and disruption of the blood-retinal 
barrier with subsequent retinal edema, as well as endothelial damage with aggravation of ischemia and non-perfusion [3, 4]. Since macular edema is a leading cause of visual impairment in patients with BRVO, various management strategies targeted towards its underlying inflammatory mediators. Grid macular laser has been commonly used for the treatment of BRVOassociated CME with mixed visual outcomes [5]. More recently, intravitreal triamcinolone acetonide or intravitreal injections of antibodies targeting VEGF are used in the management of BRVO-associated CME in an effort to improve visual outcomes [2]. However, macular edema sometimes recurs despite treatment with anti-VEGF therapy.

We reported a patient with a dramatic and rapid resolution of CME after a single injection of concurrent intravitreal Avastin (Genentech, bevacizumab $1.25 \mathrm{mg}$ ) and Triesence (Alcon, triamcinolone acetonide $2 \mathrm{mg}$ ), which was resistant to grid photocoagulation and anti-VEGF therapy.

\section{CASE PRESENTATION}

An 82-year-old female with a history of bilateral pseudophakia, gout, and hyperlipidemia using simvastatin, presented to the Loma Linda University Eye Institute due to a 4-day history of poor vision in the right eye associated with distortion in the inferior visual field. Best-corrected visual acuity (BCVA) were 20/40+2 and 20/25-2 in the right (OD) and left (OS) eyes, respectively. Examination of pupils, confrontational visual fields (CVF), and extraocular motility (EOM) was unremarkable. Slit lamp examination (SLE) showed anterior capsular phimosis in the right eye. Fundoscopy revealed a BRVO associated with macular edema and intraretinal hemorrhages along the superior arcade in the right eye (Figure1). Ocular Coherence Tomography (OCT; Heidelberg Engineering Inc., Heidelberg, Germany) of the macula revealed $\mathrm{CME}$ with intraretinal cysts involving the superior aspect of the fovea with Central Foveal Thickness (CFT) of $368 \mu \mathrm{m}$. One week later, BCVA in the right eye declined to 20/70+1 and OCT demonstrated worsening of CME. Fluorescein angiography (FA) revealed delayed venous filling of the superior venous arcade with overlying leakage, capillary non-perfusion, and cystoid macular edema (Figure 2B). The patient was treated with monthly intravitreal Avastin $(1.25 \mathrm{mg} / 0.05 \mathrm{~mL})$ for three months. In addition, grid focal macular laser was applied; laser power was titrated to produce light white-gray burns (the maximum power of 180 miliwatts with a spot size of 50 microns and duration of 100 miliseconds) in areas of nonperfusion to improve the macular edema.

However, BCVA in the right eye continued to decline and was 20/100 at the 12-week follow-up. OCT revealed continued worsening of cystoid macular edema in the right eye with CFT of $764 \mu \mathrm{m}$ (Figure 2A). After obtaining an ethical approval, concurrent intravitreal bevacizumab and intravitreal triamcinolone acetonide, was administered in the right eye. Five weeks after therapy, complete resolution of cystoid macular edema was noted; OCT demonstrated CFT improvement from $764 \mu \mathrm{m}$ to $253 \mu \mathrm{m}$ (Figure 3). BCVA in the right eye improved to 20/20-1 at 6-month follow-up.

\section{DISCUSSION}

Cystoid macular edema is a major cause of poor vision in patients with a BRVO. Laser photocoagulation remains a safe therapy, but visual outcomes have been delayed compared to anti-VEGF therapy or results might be unsatisfactory $[2,4]$. However, there is insufficient evidence illustrating the benefit of intravitreal anti-VEGF agents over grid laser photocoagulation in macular edema associated with BRVO [6]. In our case, we modified previously reported approaches in the treatment of BRVO-associated CME due to inadequate response to conventional treatment with anti-VEGF therapy coupled with macular grid photocoagulation. We concurrently administered intravitreal bevacizumab and triamcinolone acetonide, in an effort to successfully resolve the macular edema. Complete resolution of the CME and significant improvement of the vision was accomplished without any associated post-procedure complications or recurrence. These results indicate that this method is an encouraging approach to the treatment of recalcitrant CME secondary to BRVO.

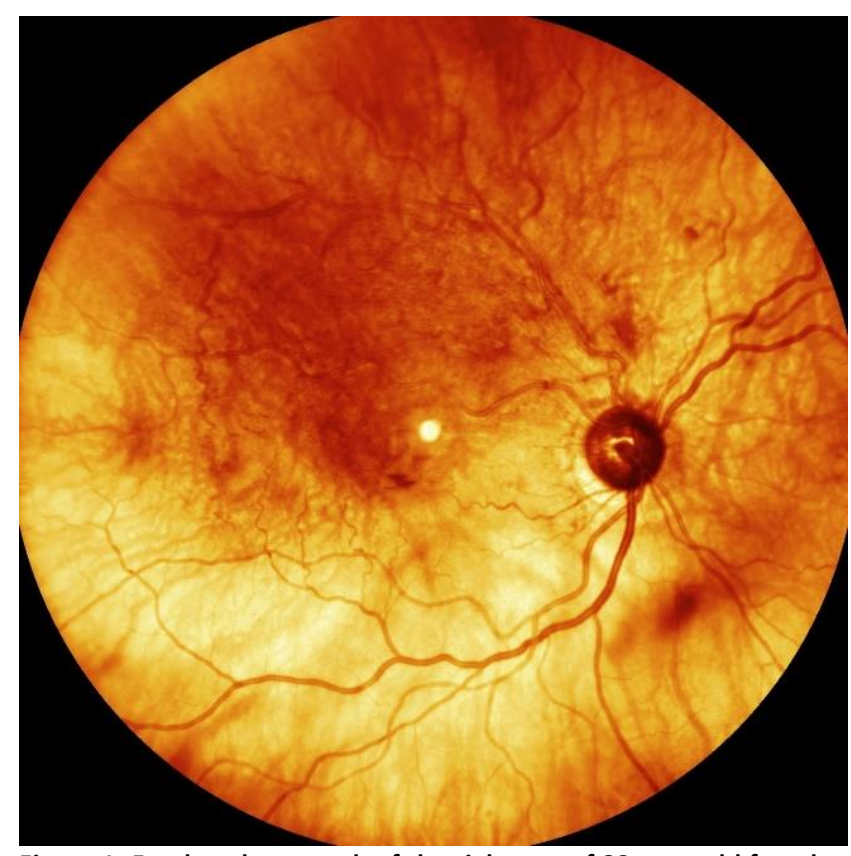

Figure 1: Fundus photograph of the right eye of 82-year-old female showing superior temporal branch vein occlusion with macular edema and multiple intraretinal hemorrhages. Tortuous retinal veins has been noted. 


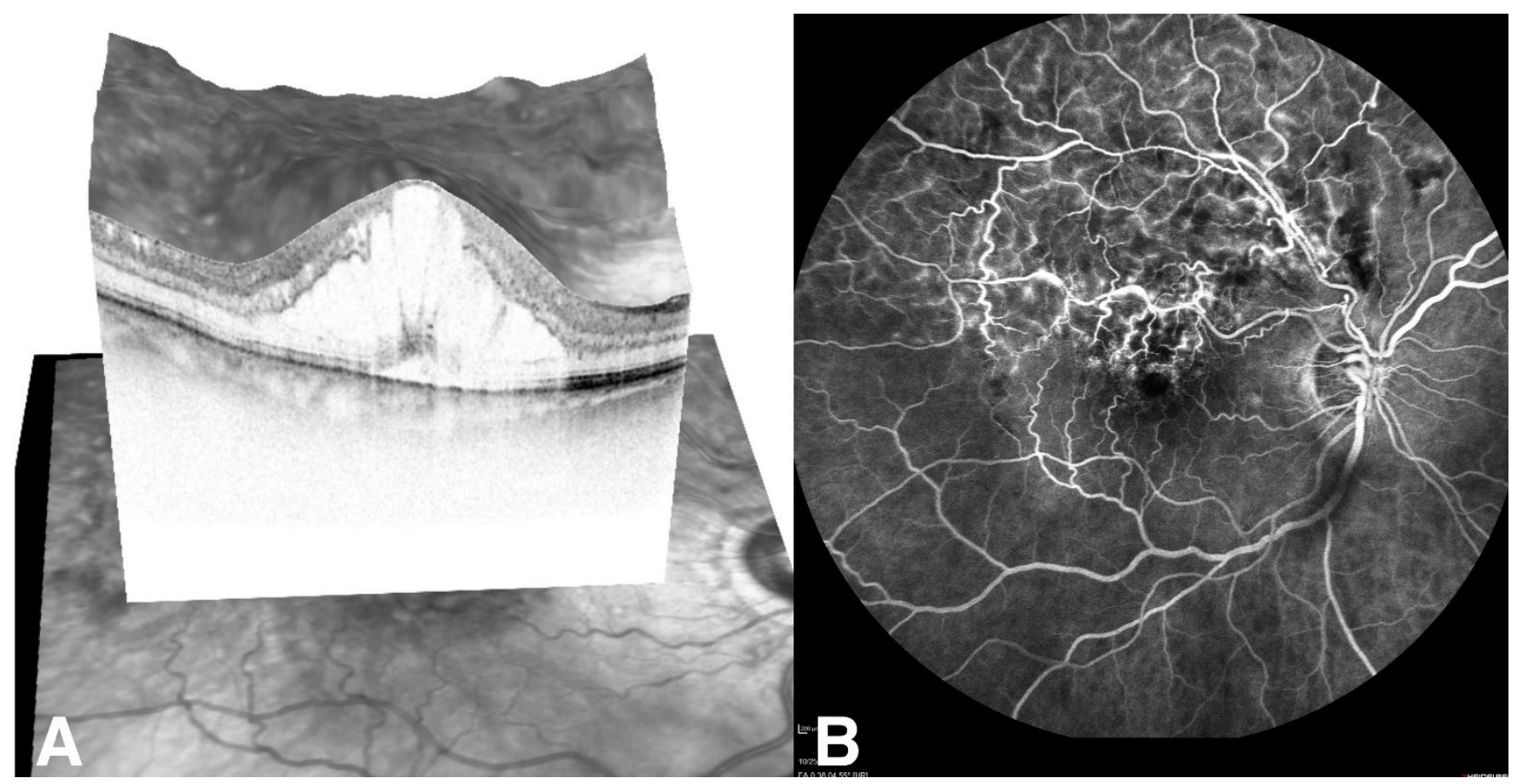

Figure 2: Before concurrent treatment with intravitreal bevacizumab and triamcinolone acetonide. A) 3-D ocular coherence tomography (OCT) of macula showing severe, recalcitrant cystoid macular edema. B) Late-phase fluorescein angiography (FA) showing capillary dropout and leakage along the superior venous arcade with superior cystoid macular edema.

Retinal vascular occlusion results in an acute release of inflammatory mediators such as IL-1, IL-6, MCP-1, ICAM-1, and VEGF [7]. These cytokines alter vascular permeability with resultant intraretinal edema, leukocyte infiltration and tissue remodeling. The foveal avascular zone is vulnerable and often results in clinically significant macular edema (CSME) with reduction in vision. Goals of management include alleviation of hypoxia, neutralization of VEGF and inhibition of production of inflammatory cytokines [2].

Grid macular photocoagulation is one of the preferred treatments of BRVO-associated macular edema. Photocoagulation of photoreceptors decreases the oxygen demand of the outer retina and increases diffusion of oxygen from the choroid to the inner retina $[8,9]$. Increased oxygenation results in autoregulatory constriction of arterioles, increased intravascular resistance, and reduced hydrostatic pressure [7]. Additionally, decrease in intravascular hydrostatic pressure (Starling's hypothesis) across the capillary wall reduces the intraretinal edema. However, treatment of BRVO-associated macular edema with grid macular photocoagulation can be ineffective due to continued production of vasopermeability factors from the hypoxic retina with resultant macular edema in the setting of BRVO [7].

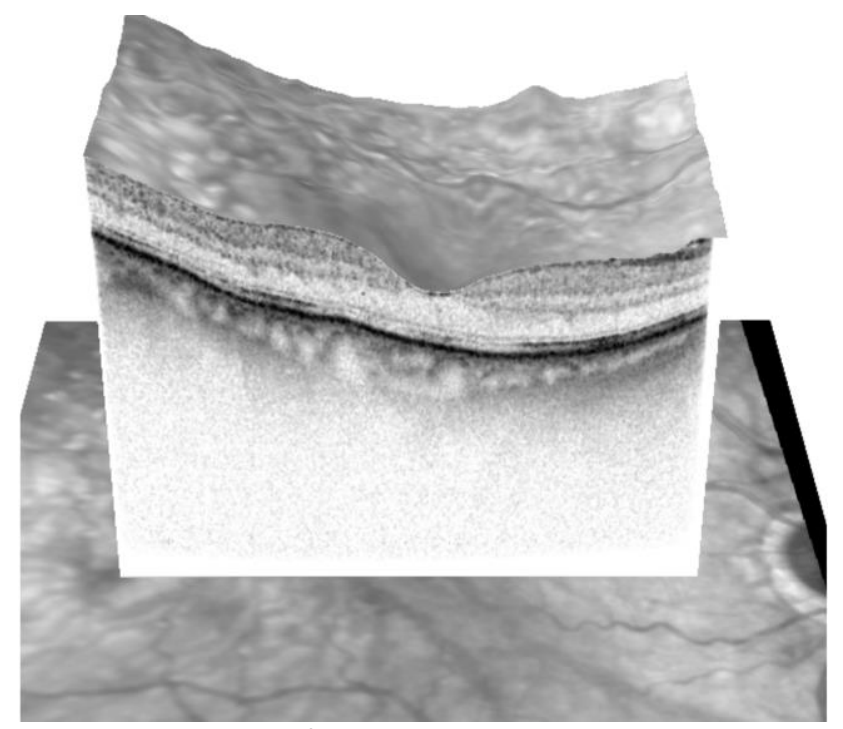

Figure 3: Five weeks after concurrent treatment with intravitreal bevacizumab and triamcinolone acetonide. 3-D ocular coherence tomography (OCT) of macula showing complete resolution of severe, recalcitrant cystoid macular edema. 
VEGF released by retinal glial and Müller cells in a hypoxic state results in alteration of tight junctions between retinal vascular endothelial cells and a consequential increase in endothelial permeability [7]. Anti-VEGF therapy is very effective in neutralizing VEGF, a common cytokine produced after an ischemic insult such as BRVO. However, it has no effect on other inflammatory cytokines such as IL-6 and ICAM-1 [4]. Aqueous levels of IL-6 are significantly elevated in BRVO compared to controls and its expression results in alteration of gap junctions and an increase in retinal vascular permeability $[4,7]$. Therefore, reducing intraocular concentrations of IL-6 and VEGF is needed for reduction of macular edema associated with BRVO. Intravitreal steroids are extremely effective in neutralizing inflammatory cytokines including IL-1, IL-6, ICAM-1 and MCP $[7,10]$.

Previous studies have demonstrated that intravitreal bevacizumab compared to intravitreal triamcinolone acetonide resulted in a similar reduction in macular edema and improvement in visual acuity in BRVOassociated macular edema [11-13]. One study reported that intravitreal injection of triamcinolone, bevacizumab, or a combination of bevacizumab and triamcinolone acetonide yielded similar therapeutic effects on macular edema at 1 month, but with intravitreal bevacizumab yielding better results in visual acuity at 6 months [14]. Other studies have reported that combined treatment with intravitreal bevacizumab and triamcinolone acetonide offered no vision advantage compared to intravitreal bevacizumab alone after 6 months [15]. We believe that concurrent administration of intravitreal triamcinolone acetonide and bevacizumab neutralized VEGF as well as all inflammatory cytokines, and promoted complete resolution of macular edema with subsequent vision improvement in our patient.

Although it was a case report, our results suggest that dual medication use including intravitreal injection of bevacizumab and triamcinolone acetonide is an encouraging and effective strategy in the treatment of BRVO-associated CME. Many studies reported traditional macular grid laser and anti-VEGF agents alone with variable outcomes; however, we provided a successful alternate treatment option for patients with BRVOassociated CME refractory to conventional interventions, which is the major strength of our study. Further randomized controlled clinical studies are necessary to determine the impact of this treatment strategy in the management of BRVO-associated CME. Overall, a balanced approach will incorporate early intervention to yield better visual and anatomic outcomes, particularly in eyes with fovea-involving macular edema.

\section{CONCLUSION}

In summary, simultaneous administration of intravitreal anti-VEGF therapy and triamcinolone acetonide resulted in a complete resolution of BRVO-associated recalcitrant macular edema, which was unresponsive to traditional treatment. This combined approach yielded a promising response and superior visual outcome in our patient. While a final consensus has not yet been reached regarding the optimal treatment algorithm for BRVOassociated $\mathrm{CME}$ resistant to established treatment patterns, medical decision should be tailored individually.

Coupling intravitreal anti-VEGF therapy with triamcinolone acetonide has a promising role in the management of CME associated with BRVO.

\section{ETHICAL DECLARATIONS}

Ethical Approval: This study conformed to the tenets of the Declaration of Helsinki. An informed consent was obtained from patient for sharing figures as well as the summary anonymously. An ethical approval has been received at the department level.

Conflict of Interest: None.

\section{FUNDING}

The author(s) received no specific funding for this work.

\section{ACKNOWLEDGEMENT}

None.

\section{REFERENCES}

1. Rogers S, Mclntosh RL, Cheung N, Lim L, Wang JJ, Mitchell P, et al. The prevalence of retinal vein occlusion: pooled data from population studies from the United States, Europe, Asia, and Australia. Ophthalmology. 2010;117(2):313-9 e1. doi: 10.1016/j.ophtha.2009.07.017 pmid: 20022117

2. Ehlers JP, Kim SJ, Yeh S, Thorne JE, Mruthyunjaya $P$, Schoenberger SD, et al. Therapies for Macular Edema Associated with Branch Retinal Vein Occlusion: A Report by the American Academy of Ophthalmology. Ophthalmology. 2017;124(9):1412-23. doi: 10.1016/j.ophtha.2017.03.060 pmid: 28551163

3. Noma H, Yasuda K, Shimura M. Cytokines and the Pathogenesis of Macular Edema in Branch Retinal Vein Occlusion. J Ophthalmol. 2019;2019:5185128. doi: 10.1155/ 2019/5185128 pmid: 31191997

4. Noma $H$, Funatsu $H$, Yamasaki $M$, Tsukamoto $H$, Mimura $T$, Sone $\mathrm{T}$, et al. Pathogenesis of macular edema with branch 
retinal vein occlusion and intraocular levels of vascular endothelial growth factor and interleukin-6. Am J Ophthalmol. 2005;140(2):256-61. doi: 10.1016/j.ajo.2005. 03.003 pmid: 16086947

5. McIntosh RL, Mohamed Q, Saw SM, Wong TY. Interventions for branch retinal vein occlusion: an evidence-based systematic review. Ophthalmology. 2007;114(5):835-54. doi: 10.1016/j.ophtha.2007.01.010 pmid: 17397923

6. Lam FC, Chia SN, Lee RM. Macular grid laser photocoagulation for branch retinal vein occlusion. Cochrane Database Syst Rev. 2015(5):CD008732. doi: 10.1002/14651858.CD008732.pub2 pmid: 25961835

7. Roy S, Kern TS, Song B, Stuebe C. Mechanistic Insights into Pathological Changes in the Diabetic Retina: Implications for Targeting Diabetic Retinopathy. Am J Pathol. 2017;187(1):919. doi: 10.1016/j.ajpath.2016.08.022 pmid: 27846381

8. Stefansson E, Landers MB, 3rd, Wolbarsht ML. Increased retinal oxygen supply following pan-retinal photocoagulation and vitrectomy and lensectomy. Trans Am Ophthalmol Soc. 1981;79:307-34. pmid: 7200671

9. Stefansson E, Machemer R, de Juan E, Jr., McCuen BW, 2nd, Peterson J. Retinal oxygenation and laser treatment in patients with diabetic retinopathy. Am J Ophthalmol. 1992;113(1):36-8. doi: 10.1016/s0002-9394(14)75750-2 pmid: 1728144

10. Sohn HJ, Han DH, Lee DY, Nam DH. Changes in aqueous cytokines after intravitreal triamcinolone versus bevacizumab for macular oedema in branch retinal vein occlusion. Acta Ophthalmol. 2014;92(3):e217-24. doi: 10.1111/aos.12219 pmid: 23889803
11. Kelkar AS, Sayyad MG, Kelkar JA, Kelkar SB, Narvankar R, Shaikh A. Comparison of single injection of intravitreal triamcinolone versus single injection of intravitreal bevacizumab in macular edema secondary to branch retinal vein occlusions with regard to changes in best corrected visual acuity and central macular thickness in the short term. Indian J Ophthalmol. 2009;57(3):243-4. doi: 10.4103/03014738.49408 pmid: 19384028

12. Guthoff R, Meigen T, Hennemann K, Schrader W. Comparison of bevacizumab and triamcinolone for treatment of macular edema secondary to branch retinal vein occlusion in a pair-matched analysis. Ophthalmologica. 2010;224(5):319-24. doi: 10.1159/000313304 pmid: 20424494

13. Byun YJ, Roh MI, Lee SC, Koh HJ. Intravitreal triamcinolone acetonide versus bevacizumab therapy for macular edema associated with branch retinal vein occlusion. Graefes Arch Clin Exp Ophthalmol. 2010;248(7):963-71. doi: 10.1007/ s00417-010-1320-2 pmid: 20182882

14. Cekic O, Cakir M, Yazici AT, Alagoz N, Bozkurt E, Faruk Yilmaz O. A comparison of three different intravitreal treatment modalities of macular edema due to branch retinal vein occlusion. Curr Eye Res. 2010;35(10):925-9. doi: 10.3109/ 02713683.2010.496540 pmid: 20858114

15. Ehrlich R, Ciulla TA, Moss AM, Harris A. Combined treatment of intravitreal bevacizumab and intravitreal triamcinolone in patients with retinal vein occlusion: 6 months of follow-up. Graefes Arch Clin Exp Ophthalmol. 2010;248(3):375-80. doi: 10.1007/s00417-009-1211-6 pmid: 19898827 